\title{
Phase Transition, Electronic and Magnetic Properties of CsN and RbN Alloys under Pressure
}

\author{
XiAO-Ping Wei ${ }^{a, *}$, YA-Ling Zhang ${ }^{b}$, Qing WANG $^{a}$ AND JiAn-Bo Deng ${ }^{c}$ \\ ${ }^{a}$ The School of Mathematics and Physics, Lanzhou Jiaotong University, Lanzhou 730070, P.R. China \\ ${ }^{b}$ Institute of Modern Physics, Chinese Academy of Sciences, 730000, Lanzhou, P.R. China \\ ${ }^{c}$ Department of Physics, Lanzhou University, Lanzhou 730000, P.R. China
}

(Received January 28, 2015; in final form July 6, 2015)

\begin{abstract}
Using full-potential local-orbital minimum-basis method within density functional theory, we study the phase transition, electronic and magnetic properties of $\mathrm{CsN}$ and $\mathrm{RbN}$ alloys under external pressure. Concerning the phase transition, we consider three possible crystal structures, including caesium chloride ( $\mathrm{CsCl}$ ), rock salt (RS) and zinc blende (ZB) ones. Calculations of enthalpy exhibit that a pressure-induced phase transition occurs between the three structures, and the phase transitions are difficult to be distinguished under ambient condition $(P=0 \mathrm{GPa})$. As the further increase of pressure, they can be specified clearly. In addition, the electronic calculations indicate that both alloys are half-metallic ferromagnets with a total magnetic moment of $2.000 \mu_{\mathrm{B}}$, which is promising for fabricating spin injection devices. Finally, we discuss the electronic and magnetic properties of CsN and RbN under external pressure. A pressure-induced delocalized electronic states and magnetic phase transition are observed in $\mathrm{RbN}$ and CsN alloys.
\end{abstract}

DOI: $10.12693 /$ APhysPolA.128.373

PACS: 71.15.Mb, 71.55.Ak, 74.62.Fj

\section{Introduction}

Half-metallic ferromagnets (HMFs), which show metallic behavior for one spin channel, while indicating semiconductor behavior for the other spin at the Fermi level, are viewed as promising materials for spintronics applications [1-3]. Recently, many compounds have been predicted to be HMF, such as magnetic oxides $\mathrm{CrO}_{2}[4,5]$ and $\mathrm{Fe}_{3} \mathrm{O}_{4}[6,7]$, perovskite $\mathrm{La}_{0.7} \mathrm{Sr}_{0.3} \mathrm{MnO}_{3}$ and $\mathrm{Sr}_{2} \mathrm{FeMoO}_{6}[8,9]$, the Heusler alloys [10-17], Mndoped GaN and Mn-doped Ge diluted magnetic semiconductors (DMSs) [18, 19], several ZB transition-metal pnictides [20-22] and chalcogenides [23]. Remarkably, HMF excluding transition metals have attracted wide attentions because of their lower stray flux with respect to traditional one.

Especially, more attention is focused on $\mathrm{CsN}$ and $\mathrm{RbN}$ alloys [24-26] owing to their potential high Curie temperature. Unfortunately, there may be several possible structures in the process of crystal growth. Therefore, it is necessary to explore the phase transition in crystal, since HMFs are as spin injection source of semiconductor only when their structure is similar with respect to one. So, we concentrate on the phase transition between $\mathrm{CsCl}$, RS, and ZB structures in present work, then argue the electronic and magnetic properties under external pressure.

The remainder of this paper is organized as follows. In Sect. 2, the method and calculation details are de-

\footnotetext{
*corresponding author; e-mail: weixp2008@gmail.com
}

scribed. In Sect. 3, the phase transition, electronic and magnetic moment of $\mathrm{CsN}$ and $\mathrm{RbN}$ alloys are presented and discussed under external pressure. Finally, some conclusions are drawn in Sect. 4.

\section{Computational method}

In this scheme, the scalar relativistic Dirac equation was solved by using full-potential local-orbital minimumbasis code (FPLO) [27, 28]. The generalized gradient approximation (GGA) was used to treat the exchangecorrelation potential [29]. The site-centered potentials and densities were expanded in spherical harmonic contributions up to $\ell_{\max }=12$. Accurate Brillouin zone integrations were performed using the standard special $k$ point technique of the tetrahedron. 204 irreducible $k$ points in the Brillouin zone are used to get the converged properties. For a self-consistent field iteration, the convergence criteria were set to both the density $\left(10^{-6}\right.$ in code specific units) and the total energy $\left(10^{-8} \mathrm{Ha}\right)$.

\section{Results and discussions}

\subsection{Equilibrium properties}

In order to explore the phase transition of $\mathrm{CsN}$ and $\mathrm{RbN}$ alloys, initially we consider three possible crystal structures, the relevant information of structures is displayed in Table I. Based on three structures, we calculate the total energy as a function of lattice constant which is shown in Fig. 1, it can be seen that RS structure is the most stable of others. It is noticeably different from the results [26], which may be ascribed to the used method and $k$ point. Moreover, we achieve the equilibrium by fitting the obtained data to the Murnaghan equation [30]. 


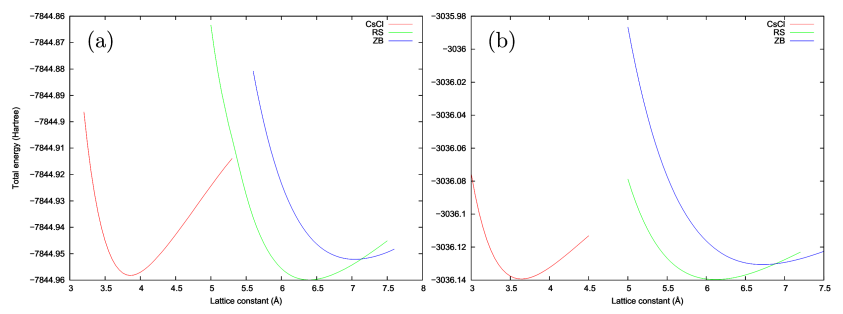

Fig. 1. The total energies per formula unit as a function of lattice constants are shown for (a) CsN and (b) RbN alloys.

Accordingly, some calculated physical parameters, such as lattice parameter, bulk modulus and the first order derivative of the modulus on volume are listed in Table II. In addition, we also give the previous results for comparison, it is found that our results are consistent with previous ones [24]. Especially, the meanfield approximation (MFA) is applied to model the Curie temperature [31], it is found that both alloys have an above-room Curie temperature, it is favorable in realistic applications.

TABLE I

The space groups and the atomic positions in the three possible phases of $\mathrm{CsN}$ and $\mathrm{RbN}$ alloys.

\begin{tabular}{c|c|c|c}
\hline \hline Phase & Space group & Cs(Rb)-position & N-position \\
\hline CsCl & $\sharp 221$ & $(0.0,0.0,0.0)$ & $\left(\frac{1}{2}, \frac{1}{2}, \frac{1}{2}\right)$ \\
RS & $\sharp 225$ & $(0.0,0.0,0.0)$ & $\left(\frac{1}{2}, \frac{1}{2}, \frac{1}{2}\right)$ \\
ZB & $\sharp 216$ & $(0.0,0.0,0.0)$ & $\left(\frac{1}{4}, \frac{1}{4}, \frac{1}{4}\right)$
\end{tabular}

TABLE II

Calculated equilibrium lattice parameters $a_{0}$ (in $\AA$ ), bulk modulus $B$ (in $\mathrm{GPa}$ ), the first order derivative of the modulus on volume $B^{\prime}$, cohesive energy $E_{\text {coh }}$ (in $\mathrm{eV}$ unit), gap $E_{\mathrm{g}}$ (in $\mathrm{eV}$ unit) and Currie temperature $T_{\mathrm{C}}$ (in $\mathrm{K}$ unit) are shown for $\mathrm{CsN}$ and $\mathrm{RbN}$ alloys, respectively.

\begin{tabular}{c|c|c|c|c|c|c}
\hline \hline \multirow{2}{*}{ Alloys } & \multicolumn{3}{|c|}{ CsN } & \multicolumn{3}{c}{ RbN } \\
\cline { 2 - 7 } & CsCl & RS & ZB & CsCl & RS & ZB \\
\hline \multirow{2}{*}{$a_{0}$} & 3.865 & 6.400 & 7.050 & 3.659 & 6.103 & 6.701 \\
& $3.69^{a}$ & $6.11^{a}$ & $6.74^{a}$ & $3.52^{a}$ & $5.92^{a}$ & $6.55^{a}$ \\
$B$ & 24.2 & 13.9 & 10.3 & 42.3 & 19.4 & 15.4 \\
$B^{\prime}$ & $23.8^{a}$ & $18.3^{a}$ & $11.3^{a}$ & $25.5^{a}$ & $17.5^{a}$ & $12.9^{a}$ \\
$E_{\text {coh }}$ & 4.70 & 3.53 & 3.05 & 3.54 & 3.05 & 2.70 \\
$E_{\mathrm{g}}$ & 4.996 & 3.043 & 2.830 & 3.107 & 3.116 & 2.869 \\
$T_{\mathrm{C}}$ & 1118.2 & 4.263 & 3.545 & 4.732 & 4.391 & 3.756 \\
\hline
\end{tabular}

${ }^{a}$ Ref. [24].

By the analysis of electronic and magnetic properties on equilibrium, we find that both alloys are halfmetallic ferromagnets with a integer magnetic moment of $2.000 \mu_{\mathrm{B}}$, and there exists a large gap in majorityspin states for their possible structures. In Table III, we have tabulated the total and atomic magnetic moment for both alloys, and noticed that magnetic moment is mainly carried by $\mathrm{N}$ atom. $\mathrm{Cs}$ and $\mathrm{Rb}$ atoms have little contribution to total magnetic moment due to their lower exchange field. For the $\mathrm{Cs}$ and $\mathrm{Rb}$ atoms, minorityspin is more than majority-spin in occupied states, thus resulting in a negative spin magnetic moment and vice versa. But a positive spin magnetic moment is observed for $\mathrm{N}$ atom. Referred to the gap, it chiefly ascribes to exchange splitting of $\mathrm{N}-p$ states in crystal field.

TABLE III

Calculated total and atomic magnetic moments of CsN and $\mathrm{RbN}$ alloys are shown under $\mathrm{CsCl}$, RS and $\mathrm{ZB}$ structures, respectively.

\begin{tabular}{c|c|c|c|c|c|c}
\hline \hline \multirow{2}{*}{ Alloys } & \multicolumn{3}{|c|}{ CsN } & \multicolumn{3}{c}{ RbN } \\
\cline { 2 - 7 } & Cs & $\mathrm{N}$ & Total & $\mathrm{Rb}$ & $\mathrm{N}$ & Total \\
\hline CsCl & -0.037 & 2.037 & $2.000\left(2.00^{a}\right)$ & -0.031 & 2.031 & $2.000\left(2.00^{a}\right)$ \\
$\mathrm{RS}$ & -0.032 & 2.032 & 2.000 & -0.057 & 2.057 & 2.000 \\
$\mathrm{ZB}$ & -0.036 & 2.036 & $2.000\left(2.00^{a}\right)$ & -0.063 & 2.063 & $2.000\left(2.00^{a}\right)$ \\
\hline
\end{tabular}

\subsection{Phase transition}

To illustrate the phase transition, we first display the relation between the pressure and lattice parameter in Fig. 2, it is more appropriate to compare with experimental settings. The pressure is expressed by the following formula:

$$
p(V)=\frac{B_{0}}{B^{\prime}}\left[\left(\frac{V_{0}}{V}\right)^{B^{\prime}}-1\right] .
$$

Regarding the phase transition, we know that the quantities of phase transition are measured by the Gibbs free energy

$$
G(P, T)=H(P, T)-T S(P, T),
$$

where the pressure $P$ and $T$ are the independent variables. The enthalpy $H$ is given by

$$
H=E+P V \text {. }
$$

At temperature $T=0$, the condition for the stable structure at constant pressure $P$ is that enthalpy be minimum. The transition pressure is really the pressure where the enthalpies of both structures are equal. Additionally, one also can determine transition pressure by calculating $E(V)$ and using the Gibbs construction of tangent lines between the $E(V)$ curves for two phases, the slope of which is the pressure for the transition between the phases. In present calculations, we exhibit the relation between enthalpy and pressure in Fig. 3. It is evident that there are three pressure-induced phase transitions in the magnetic case.

In Table IV, we have tabulated the point of phase transition. It is noted that the phase transition of $\mathrm{CsN}(\mathrm{RbN})$ occurs from $\mathrm{RS}$ to $\mathrm{CsCl}$ at $0.21(0.05) \mathrm{GPa}$; from $\mathrm{ZB}$ to $\mathrm{RS}$ at $-1.74(-1.81) \mathrm{GPa}$; from $\mathrm{ZB}$ to $\mathrm{CsCl}$ at -0.45 $(-0.70) \mathrm{GPa}$. Generally, the negative pressure means that we have to expand the $\mathrm{ZB}$ lattice to obtain the $\mathrm{CsCl}$ 


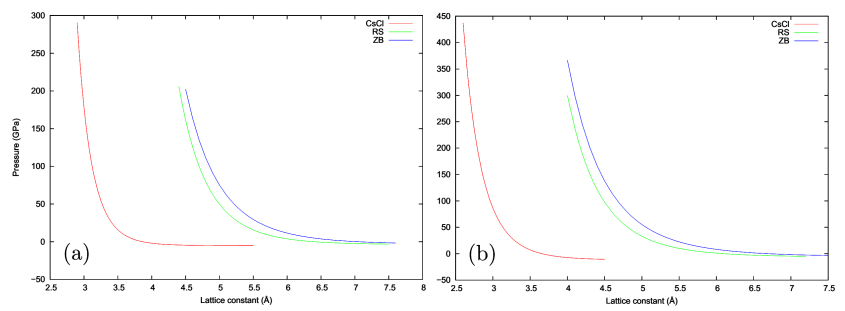

Fig. 2. The relation between lattice constant and pressure is shown for (a) CsN and (b) RbN alloys.

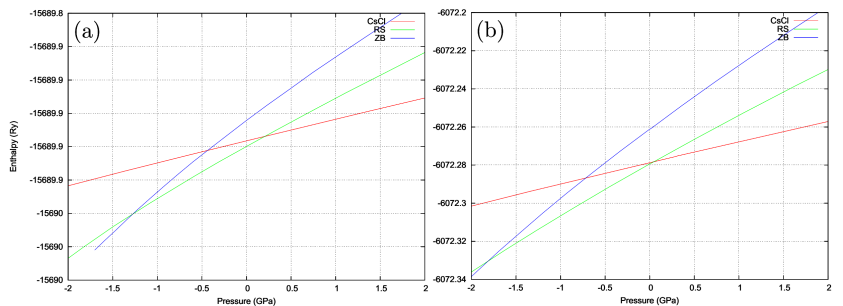

Fig. 3. Enthalpies of (a) CsN and (b) RbN alloys as a function of pressure is presented.

or RS structure. In addition, it is reminded that three structures are unable to be clearly identified due to their closed enthalpy. However, they can be distinguished as the further increase of pressure.

TABLE IV

Pressure-induced phase transition in $\mathrm{CsN}$ and $\mathrm{RbN}$ alloys. Note that $P_{\mathrm{t}}$ represents the transition pressure.

\begin{tabular}{c|c|c|c}
\hline \hline Approx. & $\begin{array}{c}P_{\mathrm{t}}(\mathrm{ZB} \longrightarrow \mathrm{RS}) \\
{[\mathrm{GPa}]}\end{array}$ & $\begin{array}{c}P_{\mathrm{t}}(\mathrm{RS} \longrightarrow \mathrm{CsCl}) \\
{[\mathrm{GPa}]}\end{array}$ & $\begin{array}{c}P_{\mathrm{t}}(\mathrm{ZB} \longrightarrow \mathrm{CsCl}) \\
{[\mathrm{GPa}]}\end{array}$ \\
\hline $\mathrm{CsN}$ & -1.74 & 0.21 & -0.45 \\
$\mathrm{RbN}$ & -1.81 & 0.05 & -0.70
\end{tabular}

\subsection{Influence of pressure on electronic and magnetic properties}

Since the density of states (DOS) of both alloys are strongly dependent on the pressure. Therefore, the dependences of moment-pressure are precisely illustrated in Fig. 4. It can see clearly that the total magnetic moments remain to be integer when pressure is close to 105.13 (145.37) $\mathrm{GPa}$ in $\mathrm{CsCl}, 15.95$ (38.64) $\mathrm{GPa}$ in $\mathrm{RS}$, 26.33 (52.46) $\mathrm{GPa}$ in $\mathrm{ZB}$ for $\mathrm{CsN}(\mathrm{RbN})$ alloy. Namely, $\mathrm{CsN}$ and $\mathrm{RbN}$ can preserve their half-metallic character within above-mentioned pressure. In addition, the dependences of volume-magnetic moment on $\mathrm{RbN}$ are also discussed in literature [32], the agreement is obtained.

Furthermore, we discuss the pressure-induced magnetic transition for CsN and RbN alloys. For CsN alloy, magnetic moment changes from 2.000 to $1.998 \mu_{\mathrm{B}}$ when the pressure is added to about $110.25 \mathrm{GPa}$, indicating a first-order magnetic transition. At $276.27 \mathrm{GPa}$, the magnetic moment becomes zero, which implies the second order magnetic phase transition from ferromagnetic

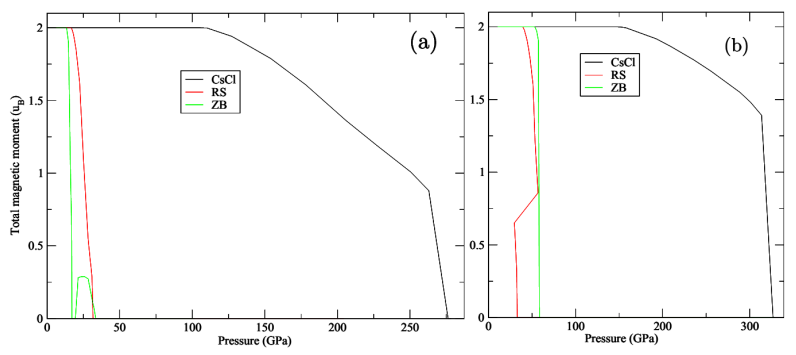

Fig. 4. It is shown the relation between total magnetic moments and pressure for (a) CsN and (b) RbN alloys.

to nomagnetic state for $\mathrm{CsCl}$ structure; the values are 16.45 GPa and 30.88 GPa for RS structure, respectively. Especially, the fist-order magnetic phase transition occur at $13.4 \mathrm{GPa}$, and then up to zero at $16.76 \mathrm{GPa}$, the magnetic moment appears again when the pressure closes to $21.33 \mathrm{GPa}$ and disappears finally at $32.88 \mathrm{GPa}$. For $\mathrm{RbN}$ alloy, the first-order transition occur at $151.31 \mathrm{GPa}$ and the second-order transition occurs at $313.58 \mathrm{GPa}$ for $\mathrm{CsCl}$ structure; $39.43 \mathrm{GPa}$ and $32.36 \mathrm{GPa}$ for RS structure; $53.34 \mathrm{GPa}$ and $5.73 \mathrm{GPa}$ for $\mathrm{ZB}$ structure, respectively. This means that the stabilities of magnetism for both alloys decrease as the increase of external pressure. Essentially, the magnetic free energy of these systems decides the first order phase transition.
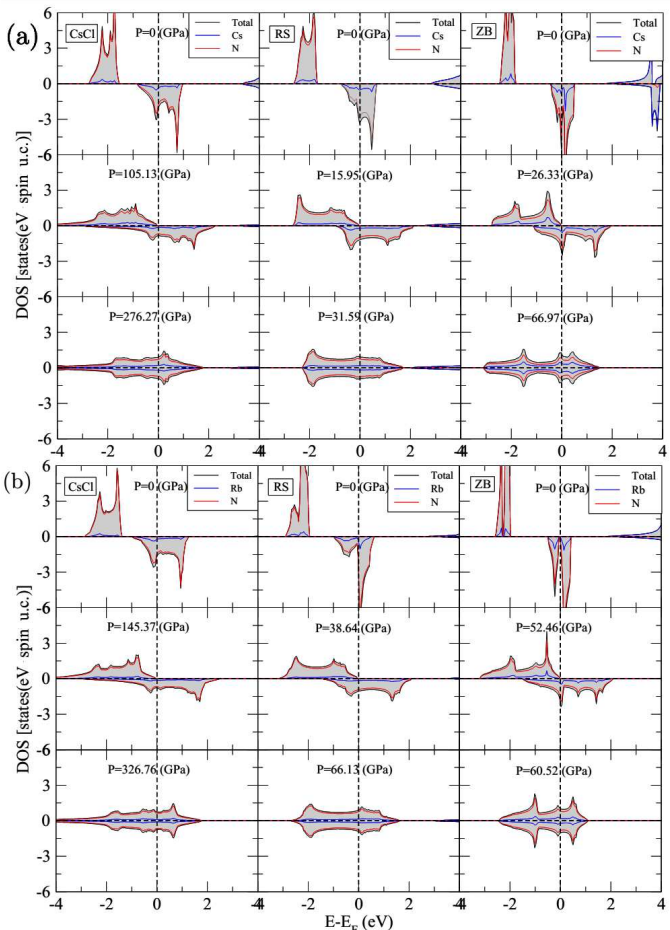

Fig. 5. Total and atomic density of states (DOS) are displayed under external pressure for (a) CsN and (b) RbN alloys.

Below we will mainly concentrate on discussing electronic properties of $\mathrm{CsN}$ and $\mathrm{RbN}$ under external pressure, because the shape of the DOS has a connection 
with the order of the quantum phase transition, thus it is necessary to give some hints about the order of the transition based on our calculations of electronic structures. In Fig. 5, we display the DOS with different pressures. We can see that N- $p$ states mainly contribute to the total density of states around the Fermi level, which means the magnetic moment is mainly carried by $\mathrm{N}$ atoms as is confirmed. The $s$ states of $\mathrm{Cs}$ or Rb give a little contribution in the vicinity of the Fermi level. A $s-p$ hybridization occurs around the Fermi level. The peaks of DOS in minority-spin states (van Hove singularities) near the Fermi level result in a ragged free energy landscape under equilibrium state, resulting in the occurrence of first order phase transition. The $s$ and $p$ states are delocalized with the further increase of pressure, and the Fermi level slowly shifts to the edge of valence band maximum and finally pass through it, the related physical nature is also changed gradually from half-metallic to metallic characters.

\section{Conclusion}

By using electronic structure calculations based on density functional theory, we study the effect of pressure on phase transition, electronic and magnetic properties of $\mathrm{CsN}$ and $\mathrm{RbN}$ with $\mathrm{CsCl}, \mathrm{RS}$ and $\mathrm{ZB}$ structures. Both of alloys are half-metallic ferromagnets and have an integer magnetic moment of $2.000 \mu_{\mathrm{B}}$. Pressure-induced the phase transition is systematically studied. In addition, we observe that the three structures are unable to be clearly identified at their equilibrium due to their closed enthalpy. However, they can be distinguished as the further increase of pressure. For the electronic and magnetic properties under external pressure, it is found that the first-order and second-order magnetic transitions occur as further increase of pressure, respectively. Simultaneously, the electronic states are delocalized with the increase of pressure, and the Fermi level slowly shifts to the edge of valence band maximum and finally passes through it, the related physical nature is also changed gradually from half-metallic to metallic characters. In summary, the study may provide some valuable hints for the fabrication of spintronic devices.

\section{Acknowledgments}

The Project is supported by the Young Scholars Science Foundation of Lanzhou Jiaotong University (No. 2015028). Discussions with W.X. Zhang are greatly acknowledged.

\section{References}

[1] R.A. de Groot, F.M. Mueller, G.P. van Engen, K.H.J. Buschow, Phys. Rev. Lett. 50, 2024 (1983).

[2] H. Ohno, Science 281, 951 (1998).

[3] T. Graf, C. Felser, S.S.P. Parkin, Prog. Solid State Chem. 39, 1 (2011).
[4] K. Schwarz, J. Phys. F Met. Phys. 16, L211 (1986).

[5] M.A. Korotin, V.I. Anisimov, D.I. Khomskii, G.A. Sawatzky, Phys. Rev. Lett. 80, 4305 (1998).

[6] A. Yanase, K. Siratori, J. Phys. Soc. Jpn. 53, 312 (1984).

[7] S. Soeya, J. Hayakawa, H. Takahashi, K. Ito, C. Yamamoto, A. Kida, H. Asano, M. Matsui, Appl. Phys. Lett. 80, 823 (2002).

[8] Y.P. Liu, S.H. Chen, J.C. Tung, Y.K. Wang, Solid State Commun. 152, 968 (2012).

[9] K.-I. Kobayashi, T. Kimura, H. Sawada, K. Terakura, Y. Tokura, Nature 395, 677 (1998).

[10] G.D. Liu, X.F. Dai, H.Y. Liu, J.L. Chen, Y.X. Li, G. Xiao, G.H. Wu, Phys. Rev. B 77, 014424 (2008).

[11] K. Özdog̃an, E. Şaşıoglu, B. Aktaş, I. Galanakis, Phys. Rev. B 74, 172412 (2006).

[12] J. Li, G.F. Chen, H.Y. Liu, Y.X. Li, J.F. Li, X.W. Xu, J. Magn. Magn. Mater. 322, 1 (2010).

[13] N. Kervan, S. Kervan, Intermetallics 24, 56 (2012).

[14] A. Birsan, P. Palade, V. Kuncser, Solid State Commun. 152, 2147 (2012)

[15] H.Z. Luo, G.D. Liu, F.B. Meng, J.Q. Li, E.K. Liu, G.H. Wu, J. Magn. Magn. Mater. 324, 3295 (2012).

[16] H.M. Huang, S.J. Luo, K.L. Yao, J. Magn. Magn. Mater. 324, 2560 (2012)

[17] X.P. Wei, J.B. Deng, G.Y. Mao, S.B. Chu, X.R. Hu, Intermetallics 29, 86 (2012).

[18] L. Kronik, M. Jain, J.R. Chelikowsky, Phys. Rev. B 66, 041203(R) (2002).

[19] G. Liu, B.G. Liu, Phys. Rev. B 73, 045209 (2006).

[20] I. Galanakis, P. Mavropoulos, Phys. Rev. B 67, 104417 (2003).

[21] B.G. Liu, Phys. Rev. B 67, 172411 (2003).

[22] Z.Y. Chen, B. Xu, G.Y. Gao, J. Magn. Magn. Mater. 347, 14 (2013).

[23] W.H. Xie, Y.Q. Xu, B.G. Liu, D.G. Pettifor, Phys. Rev. Lett. 91, 037204 (2003).

[24] A. Lakdja, H. Rozale, A. Chahed, Comp. Mater. Sci. 67, 287 (2013).

[25] X.P. Wei, Y.D. Chu, X.W. Sun, Y. E, J.B. Deng, Y.Z. Xing, J. Magn. Magn. Mater. 363, 55 (2014).

[26] A. Lakdja, Comp. Mater. Sci. 89, 1 (2013).

[27] K. Koepernik, H. Eschrig, Phys. Rev. B 59, 1743 (1999).

[28] I. Opahle, K. Koepernik, H. Eschrig, Phys. Rev. B 60, 14035 (1999).

[29] J.P. Perdew, K. Burke, M. Ernzerhof, Phys. Rev. Lett. 77, 3865 (1996).

[30] F.D. Murnaghan, Finite Deformation of an Elastic Solid, Dover, New York 1967.

[31] Ph. Kurz, G. Bihlmayer, S. Blügel, J. Phys. Condens. Matter 14, 6353 (2002).

[32] K. Zberecki, L. Adamowicz, M. Wierzbicki, Phys. Status Solidi B 246, 2270 (2009). 